\title{
Eficacia comunicativa en el habla infantil. Indicadores pragmá- ticos básicos
}

\author{
Milagros Fernández Pérez \\ Catedrática de Lingüística general \\ Universidade de Santiago de Compostela \\ Facultad de Filología \\ Campus Norte - Burgo das Nacións \\ 15782 Santiago de Compostela (Galicia) \\ E-mail: magos.fernandez.perez@usc.es
}

\section{EFICACIA COMUNICATIVA EN EL HABLA INFANTIL. INDICADORES PRAGMÁTICOS BÁSICOS}

\begin{abstract}
RESUMEN: En este trabajo se subraya la eficacia comunicativa como ingrediente destacado para valorar el habla infantil. Tal es su relevancia que las dinámicas adquisitivas de la lengua se construyen esencialmente sobre los componentes que definen dicha eficiencia. Así que el cometido específico de esta contribución es constatar el papel de cuatro indicadores pragmáticos que regulan la potencia comunicativa básica en la interacción infantil, y que son: (a) intenciones comunicativas; (b) respuesta a la comunicación; (c) interacción y conversación, y (d) variación verbal según el contexto (Dewart \& Summers 1988). Estos cuatro parámetros incidentes en niveles de éxito de los intercambios constituyen asimismo elementos decisivos para estimar la adquisición en las primeras etapas, más allá de posibles requisitos sobre componentes y estructuras gramaticales. El lenguaje infantil es una evoluciónen-proceso no equivalente a la lengua adulta conformada, de modo que la "gramática del uso" en la interacción es la que se erige como plataforma sobre la que paulatinamente se irá construyendo la gramática formal (Givon 2002, 2009).
\end{abstract}

PALABRAS CLAVE: Comunicación infantil; Eficacia en el habla infantil.

SUMARIO: 1. Introducción. Fundamentos de la comunicación infantil. 2. Condicionantes pragmáticos básicos de eficacia comunicativa. 3. Eficacia comunicativa y evaluación del lenguaje infantil. 4. Conclusión. La eficacia comunicativa como pauta de desarrollo verbal.

\section{COMMUNICATIVE EFFEC- TIVENESS IN CHILD LAN- GUAGE. BASIC PRAGMATIC INDICATORS}

ABSTRACT: This paper highlights communicative efficacy as a prominent ingredient for evaluation of child language. Such is its relevance that the dynamics of language acquisition are built largely on the components that define the effectiveness in child language. So, the specific task of this paper is to argue for the role of four pragmatic markers that regulate the basic communicative strength in child interaction, namely: (a) communicative intentions; (b) response to communication; (c) interaction and conversation, and (d) contextua variation (Dewart \& Summers 1988). These four parameters are also decisive elements for the evaluation of language acquisition in the early stages, beyond possible prerequisites related to grammatical components and structures. Child language is a work-in-progress, not comparable to adult language. Which means that "usage grammars" in interactions constitute the basis over which the formal grammar shall be built (Givon 2002, 2009).

KEY WORDS: Child communication; Effectiveness in child language.

SUMMARY: 1 . Introduction Fundamentals on child communication. 2. The basic settings of effectiveness in child language. 3. Communicative effectiveness and the child language evaluation. 4. Conclusion. The communicative effectiveness as a guideline of the verbal development.

\section{EFFICACITE COMMUNICATIVE DANS LA PAROLE DES ENFANTS. DES INDICATEURS PRAGMA- TIQUES BASIQUES}

RÉSUMÉ : Dans ce travail on souligne l'efficacité communicative comme facteur évaluant la parole des enfants. Les dynamiques d'acquisition du langage ont lieu autour des composants qui définissent cette efficacité. L'objectif de cette contribution est de vérifier la fonction des quatre indicateurs pragmatiques qui régulent le potentiel communicatif dans les interactions des enfants, à savoir : (a) intentions communicatives ; (b) réponse à la communication ; (c) interaction et conversation ; et (d) variation d'après le contexte (Dewart \& Summers 1988). Ces quatre paramètres ayant une incidence sur le succès des interactions verbales sont aussi des éléments décisifs pour apprécier l'acquisition du langage dans les premières étapes, au-delà des conditions autour des composants et des structures grammaticales. La parole des enfants est une dynamique évolutive et en processus qui n'est pas équivalente à la parole adulte définie, de sorte que la " grammaire de l'usage " est le fondement sur lequel on construit progressivement la grammaire formelle (Givon 2002, 2009).

MOTS CLÉS: Communication des enfants; Efficacité dans la parole des enfants.

SOMMAIRE: $1 . \quad$ Introduction. Fondaments de la communication infantile. $2 . \quad$ Conditions pragmatiques essentielles de l'efficacité communicative. 3. L'efficacité communicative et l'évaluation du langage des enfants. 4. Conclusion. L'efficacité communicative comme modèle de développement verbal. 


\title{
Eficacia comunicativa en el habla infantil. Indicadores pragmá- ticos básicos ${ }^{1}$
}

\author{
Milagros FernÁNDEZ PÉREZ
}

\section{INTRODUCCIÓN. FUNDAMENTOS DE LA COMUNICACIÓN INFANTIL}

La disposición comunicativa incluso en bebés de pocos meses es algo admitido desde hace ya cierto tiempo. Tanto que se ha desterrado el calificativo de prelingüistico aplicado al período inicial de desarrollo y en su lugar se habla de fase preidiomática. El animal comunicativo por excelencia que es la especie humana —el homo loquens que por naturaleza somos- perfila muy pronto sus capacidades intersubjetivas primarias (Trevarthen 1980, 1993, 1998). Ya en el nivel molar o reactivo el niño solicita atención y establece contacto y comunicación a través de los sentidos, desde el nacimiento informa sobre sus necesidades y su estado. Algunos autores como Bruner (1983) sostienen que el bebé y su madre crean un formato interactivo de predicción, una especie de microcosmos en el que se dan intercambios emocionales comunicativos. Se constata incluso un marco intencional básico que cada uno de ellos interpreta gracias al contexto en el que se producen las interacciones. Precisamente, son las intenciones las que - parececonstituyen el factor crucial para orientar los mensajes y dirigir la comunicación. En ellas descansa el dominio de la eficacia comunicativa. No en vano contemplar los intereses del interlocutor es requisito ineludible para que el proceso de interacción sea cooperativo, se produzca la empatía prevista, y la comunicación resulte exitosa.

Alrededor de las coordenadas de atención común que desde el primer momento va definiendo las interacciones, toman cuerpo las pretensiones básicas de los interlocutores. Posteriormente, y a medida que se amplian los contextos interactivos, se incrementa el dominio de intenciones. En opinión de Tomasello (2003: 22 y ss.): los niños desarrollan desde los primeros meses habilidades sociales que se plasman en una estructura dual. Se trata de dos vertientes que son fundamento y esencia para que emerjan las dimensiones simbólicas de la comunicación lingüística. De una parte, los marcos de atención común definen cimientos intersubjetivos que facilitan la comprensión de propósitos comunicativos y su posible relevancia. Los rituales a

\footnotetext{
1 Todos los ejemplos aducidos en este trabajo están extraídos del Corpus koiné de habla infantil elaborado en el área de Lingüística General de la Universidade de Santiago por el grupo del mismo nombre (www.usc.es/koine). El inventario se halla a disposición de usuarios en el sitio CHILDES-TalkBank: https://phonbank.talkbank.org/access/Spanish/Koine.html. La fuente de datos ha sido posible gracias a la financiación de distintos proyectos en convocatorias competitivas del Gobierno central y de la Xunta de Galicia desde finales de los ' 90. Particularmente, esta contribución se inscribe en el proyecto financiado por FEDER/Ministerio de Ciencia, Innovación y Universidades-Agencia Estatal de Investigación, Adquisición fónica y corpus. Tratamiento en PHON del corpus koiné de habla infantil (FFI2017-82752-P).
} 
la hora del baño o de la comida con la concurrencia repetida de gestos, miradas, o actos ejemplifican ese proceso de depósito intencional e interactivo. Que muchos casos de autismo se detecten por la ausencia de este tipo de intercambios prueba la importancia de las capacidades intersubjetivas primarias. De otra parte, las intenciones de los interlocutores adultos van tomando solidez hasta que son los niños quienes, imitando, las monitorizan (Levelt, 1983). Una expresión como "¡Un payaso!, toma", emitida por el adulto, suele ser repetida por el niño en el momento de recoger el juguete; para, en una etapa posterior, tomar la iniciativa y decir "¡Un payaso!, toma" cuando lo entrega, e incluso alternar con "iUn payaso!, trae", cuando lo recibe $^{2}$. El mismo proceso se da en aquellas situaciones en las que el adulto manifiesta un deseo para que el niño lleve a cabo una acción, "Yo quiero que la nena tome la lechen: si primero se reitera en la secuencia manteniendo la primera persona, más tarde el niño introduce su propia perspectiva y hace figurar la tercera persona, "La mamá quiere que la nena tome la leche».

Las acciones repetidas actúan como canales que paulatinamente dan sostén a las percepciones y a los procesos de cognición asociados. Las investigaciones sobre las neuronas espejo (Rizzolati \& Arbib 1998, Arbib 2002, Buccino et alii 2004, Corballis 2006) constatan a este respecto que el depósito perceptual determina y condiciona la dinámica de adquisición de la lengua, habida cuenta de que se trata de un proceso que se perfila a través de imitaciones y reiteración. En opinión de Arbib (2002: 33): "the ability to imitate is a key innovation in the evolutionary path leading to language in the human".

Las asociaciones acción-objeto incorporan no sólo la parte motora sino también el reconocimiento visual del objeto. La sistematicidad de estos marcos perceptivos deriva en la emergencia de simbolos que especifiquen las acciones, los objetos y su relación. De ahí al proceso de nombrar y al desarrollo del lenguaje hay un paso. Arbib (2002) sugiere una progresión evolutiva desde la acción (praxis pura) pasando por la pantomima (comprensión de la acción) hasta llegar al (proto) lenguaje (expresiones simbólicas). En su opinión, "The cultural evolution of Homo sapiens may then have involved an increased ability to name actions and objects to create on rapidly growing set of verb-argument structures, and the ability to compound those structures in diverse ways" (Arbib 2002: 32).

No es, pues, de extrañar que el marco perceptivo y conceptual resulte crucial para el reconocimiento de palabras. En opinión de Tomasello (2003, cap. 3): conviene distinguir tres tipos de factores en el proceso de discriminación y uso de unidades léxicas en el habla infantil. Hay factores funda-

\footnotetext{
${ }^{2}$ La repetición desempeña un papel crucial en el progreso verbal ya que incrementa la "base común" en la que participan todos los interlocutores en un intercambio. Con palabras de E. Clark (2006:79): “cette augmentation reflète l'usage accepté, quotidien, dans la communauté qui entoure l'enfant. Répéter, c'est en même temps signaler l'adoption des conventions et l'augmentation de la base commune".
} 
mentales, hay pre-requisitos, y hay elementos facilitadores. Pues bien, prestar atención reiterada a las intenciones comunicativas de los adultos es fundamental para reconocer y construir los marcos conceptuales requeridos en la dinámica facilitadora del contraste léxico. Para que el niño distinga las palabras y pase del uso de significados genéricos (de "perro" para denominar cualquier animal) a los significados específicos y particulares (a "lobo", "león", "ternero"): se requieren los contextos y la variedad de intenciones: "Language acquisition begins when it does because it depends on the ability to share attention with other humans beings communicatively and so to form symbols, an ability that emerges near the end of the first year of life" (Tomasello 2003:90-91).

Conseguir eficacia comunicativa significa lograr un determinado propósito - sea transaccional, sea puramente informativo- a través de un mensaje. Y estas expectativas están presentes en el habla de los niños. Si un bebé no recibe atención, seguirá reclamándola. Los casos de reparaciones, aclaraciones, malestar o enfado cuando los mensajes no han resultado efectivos son frecuentes en el período de desarrollo de la lengua. Desde muy pronto los niños saben discernir si sus mensajes han resultado o no exitosos, hasta el extremo de obligar a los interlocutores a entrar en un proceso de explicaciones y reformulaciones que faciliten su propósito (Clark \& Chouinard 2000).

Como telón de fondo indispensable de las dinámicas de socialización e interacción en las primeras etapas de adquisición de la lengua figuran las percepciones cognitivas (siempre proyectadas en una visión de mundo específica): las normas sociales, y los principios idiomáticos que definen un depósito de reglas codificadas. Sin duda, en estos condicionantes descansa la comunicación y su eficacia. El desarrollo conceptual ligado al entorno destaca características lingüísticas particulares. Es habitual que cuando un gemelo o un mellizo se refiere a sí mismo, lo haga en plural. La emergencia de los pronombres personales está fuertemente condicionada por el input que reciba el niño según su ubicación en el ámbito familiar: las interacciones diádicas entre hermanos facilitan la deixis, la posesión y el uso de pronombres personales (Deutsch et alii 2001). Por otra parte, son de común asumidas las repercusiones del habla dirigida y del entorno idiomático en la tónica comunicativa del habla infantil. Depende de con quien interaccionen para que en los usos predominen ciertas funciones pragmáticas o determinados valores sociales. Es normal que los niños japoneses utilicen desde muy pronto fórmulas de cortesía como "arigato" ('gracias'): o "dozo" ("por favor'). Y es también frecuente que en comunidades del Pacífico y de la Micronesia - en las que los niños no son receptores de habla dirigida ni tampoco se contemplan como sujetos que intervengan en la conversación- figuren ya en etapas iniciales de desarrollo palabras o estructuras con connotaciones sociales marcadas. En kaluli (Papúa Nueva Guinea) sólo las niñas utilizan la palabra "elema" ('se dice así...'): asociada a la función de corrección que se atribuye a las mujeres (Ochs \& Schieffelin 1995). 
El principio de que "todos los niños hablan" no es condición suficiente para abordar la efectividad comunicativa en el habla infantil (Clark 2003). La diversidad de entornos y de estímulos motiva que se definan normas y usos particulares no ajustables a la uniformidad del proceso tantas veces defendida ${ }^{3}$.

La presencia de un código compartido que facilite los intercambios es requisito indispensable para que se produzca la comunicación. Como es natural, si no hay pautas comunes, no cabe la comprensión de mensajes. Conviene no olvidar que no se trata solo de reglas en el dominio estructural sobre en qué modo organizar expresiones o en qué manera combinar unidades, sino que además y sobre todo son relevantes ciertos aspectos y comportamientos de uso que dan sentido a las emisiones ${ }^{4}$. No basta con fijar principios de gramáticas especiales o quasigramáticas (Fernández Pérez 2006a, 2006b) que contengan las singularidades del habla infantil, como pueden ser los procesos fónicos propios (por ejemplo, [láminas] para lo que posteriormente será [lágrimas]); o el papel de señalizadores referenciales que desempeñan los elementos determinantes en la lengua adulta (artículos, demostrativos, indefinidos, posesivos): como en "Ahí hay un agua"; "Arriba, mira un oro". Por el contrario, se hace previa la atención a aquellos ingredientes de contexto envolventes que son indispensables para que la comunicación resulte efectiva, pueda ser eficaz. En una palabra, que se vuelve aquí pertinente el lema de que "el discurso de hoy es la gramática de ma-

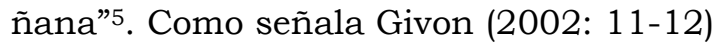

Humans can, under a variety of developmental, social or neurological conditions, communicate without grammar, using the well-coded lexicon with some rudimentary combinatorial rules (...)

The slow and analytical pre-grammatical communication is heavily vocabulary driven. This tallies with the fact that vocabulary is acquired before grammar, in both first and second language acquisition. Thus, pre-grammatical children, adult pidgin speakers

\footnotetext{
${ }^{3}$ Son abundantes las investigaciones sobre el desarrollo cognitivo y lingüístico particular de niños en diferentes entornos idiomáticos y en distintos contextos de motivación y estimulación. La importancia de lo específico ha cobrado protagonismo sobre lo universal y lo automático. El enfoque de, entre otros, los trabajos contenidos en el compendio editado por Melissa Bowerman (2001) constata que los niños no son máquinas en el proceso de desarrollo de la lengua, que las categorías del lenguaje adulto no se ajustan a las unidades del habla infantil, y que no procede asumir desarrollos cognitivos y desarrollos idiomáticos como dinámicas totalmente desconectados ni tampoco como actividades totalmente dependientes.

${ }^{4}$ En todo marco comunicativo lo ciertamente relevante es la acomodación del sentido en el contexto. Mucho más si se trata de habla peculiar como la que corresponde al período de adquisición o la que puede producirse en casos de regresión o deficitarios. Aquí, las claves del valor lingüístico de las expresiones no residen tanto en el grado de limitación estructural cuanto en su asociación funcional esperable a un contexto. No hay que olvidar que las hormas en las que se canalizan los significados están siempre sujetas a un grado notable de variabilidad, por lo que "la resolución de las diferentes opciones disponibles se hace estrechamente dependiente de los contextos o situaciones comunicativas” (Hernández Sacristán 2006:105).

${ }^{5}$ A este respecto, las consideraciones de Roger Brown (1968:288) avanzaban principios teóricos de la gramática emergente y procesual defendida por el cognitivismo: "The changes produced in sentences as they move between persons in discourse may be the richest data for the discovery of grammar".
} 
and agrammatical aphasics comprehend and produce coherent connected discourse, albeit at slower speeds and high error rates than those characteristics of grammatical language.

El denominado enfoque pragmático-social (Tomasello 2003: 87 y ss.) permite contemplar, al menos en una primera fase, el grado de eficacia comunicativa en el habla infantil. En este trabajo me limitaré a delinear qué dimensiones pragmático-sociales considero ineludibles para que se produzcan significados en las interacciones. Son una especie de indicadores que orientan la elaboración de expresiones para conseguir ciertos indices de efectividad. Como cabe sospechar, no voy a entrar en la valoración de usos sistemáticos ni tampoco en criterios evaluativos concretos de éxito comunicativo que incluyan a la gramática. Me ceñiré exclusivamente a los parámetros determinantes del sentido comunicativo y que, por tanto, son propiedades consustanciales y sine qua non de la eficacia.

\section{CONDICIONANTES PRAGMÁTICOS BÁSICOS DE EFICACIA COMUNICATIVA}

En consonancia con las disposiciones elementales de socialización $-\mathrm{y}$ que comúnmente se han etiquetado con los términos de marcos de atención común y marcos de interpretación de intenciones -, es posible reconocer cuatro dimensiones esenciales asociadas a la efectividad de los intercambios. A saber, (1) las intenciones comunicativas (sean conscientes y controladas, sean imitadas, o sean automatizadas): (2) la respuesta a la comunicación (sea o no respuesta lingüística): (3) la interacción y la conversación, y (4) la variación verbal ligada a diferentes contextos.

\subsection{INTENCIONES Y COMUNICACIÓN INFANTIL}

Resulta indiscutible que la interacción sólo se produce si se activa un intercambio, si hay interés o intención (sea o no consciente) de hacerlo. Desde muy pronto los bebés promueven la comunicación incitados por necesidades fisiológicas. $\mathrm{Y}$ ya en los primeros meses se registran intercambios comunicativos consistentes en juegos guturales, manuales o faciales. El carácter ostensivo y referencial del grueso de palabras adquiridas en las fases iniciales ilustra usos verbales interesados, con intención y propósito claros.

Las dos muestras que se ofrecen a continuación contienen elementos intencionales claros de "queja" en el caso de Xaquín (con apenas dos años) en el ejemplo 1; y de "localización" cuando hablan Borja (casi con tres años) y Martin (de tres años recién cumplidos): en el ejemplo 2 


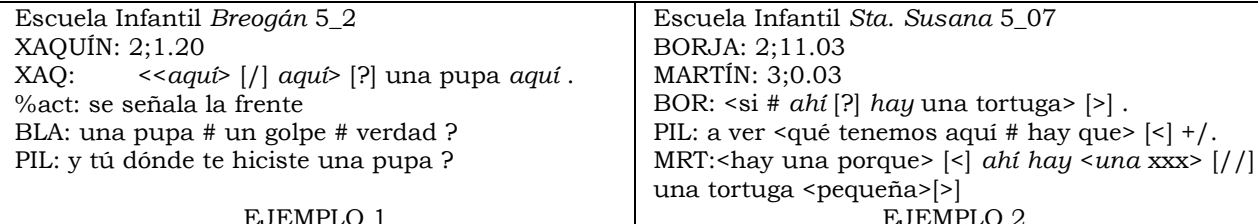

Como es natural, los verbos más frecuentes en estas etapas tempranas contienen significados intencionales marcados: "mira", "toca", "quiero", "trae", "toma", "vete". Parece ser que la dinámica de adquisición de procesos y acciones se asocia a la comprensión de intenciones en el marco de contextos definidos (Tomasello 2003: 67 y ss.). Las producciones que a continuación se relacionan confirman esta circunstancia. Irene (de apenas dos años, en el ejemplo 3) reitera su solicitud hasta hallar respuesta transaccional. También David (con poco más de tres años) y Antía (que aún no los ha cumplido): en el ejemplo 4, se apoyan intencionadamente insistiendo en su requerimiento de agua a pesar de las negativas, quizás porque ambos tienen sed:

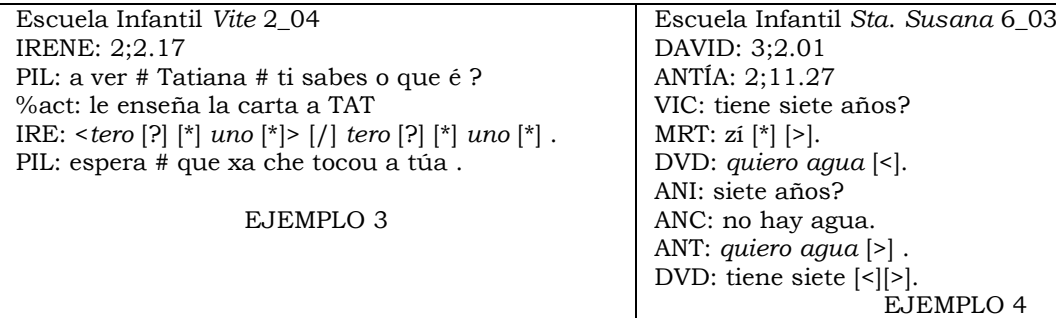

La atención hacia personas y objetos refleja deseos de interacción y desencadena señalizaciones, preguntas o reacciones. Lo que es prueba palpable de actividad comunicativa incluso en etapas iniciales y que parece ser sintoma de trastorno cuando esas inquietudes por los intercambios desaparecen ${ }^{6}$. Hay, así, comportamientos y aspectos de interacción concretos que traslucen la vivacidad intencional. Entre otros, las actitudes de rechazo y negativas, las expresiones de autoaserción, los comentarios o las apelaciones. Es el caso de la conversación entre Artai y Iago (que todavía no han cumplido los tres años, ejemplo 5): cargada de apelaciones y comentarios expresivos sobre las sensaciones que les provoca una culebra.

\footnotetext{
${ }^{6}$ Es el caso de niños con autismo o con síndrome de desarrollo del lenguaje, que se detectan por su pasividad y desinterés hacia la socialización. Hay ausencia de intenciones.
} 
Escuela Infantil Elfos 2_06

ARTAI: $2 ; 6.3$

IAGO: $2 ; 9$.

ART: $\mathrm{mi}\left[{ }^{*}\right]<$ que culeba $\left[{ }^{*}\right]$ muy rande $\left[{ }^{*}\right]>\left[{ }^{*}\right]$ \# e etá $\left[{ }^{*}\right]$ morta .

IAG: ah@i \#xxxa mata [*].

\%act: la intenta enterrar con su pala

IAG: ala \# ya maté .

ART: y ahora $\mathrm{xxx}$ ota $\left[{ }^{*}\right]$ pa [*] que $\mathrm{xxx}$.

ART: Iago \# eta [*] \# non a matex [*] \# que no ten dentex [*]

ART: $x x x$ \# é pequeniña.

El componente emotivo se vuelve crucial para la efectividad comunicativa. La función expresiva de intenciones verbalizadas es también detonante de incidencia apelativa. No hay que olvidar que la empatia es factor clave para una comunicación eficaz. De modo que, si la carga intencional es tan clara y precisa como para activar y mover al interlocutor, la fluidez del proceso está garantizada y es previsible un grado notable de efectividad y éxito en la interacción.

\subsection{RESPUESTA A LA COMUNICACIÓN}

La contestación - sea o no verbal-a los intercambios comunicativos es prueba irrefutable de habilidad para interaccionar. No sólo porque indica interés y atención hacia el emisor, sino porque precisamente es en las respuestas en donde se hallan datos sobre la comprensión de intenciones y sobre los propósitos particulares del mensaje. El tipo de input o los estímulos a los que el niño reacciona, así como la naturaleza de las respuestas son elementos clave para comprobar su nivel de participación comunicativa. En el ejemplo que se proporciona a continuación, es Artai (de casi cuatro años) quien toma la iniciativa y decide que sea Iago (con dos meses más) el que narre el cuento. Y éste, por su parte, asume el papel protagonista tratando de clarificar los dibujos con matices sobre sus trazos y perfiles: se pregunta si en una viñeta hay un coche, se sorprende de que el sol no tenga rayos.

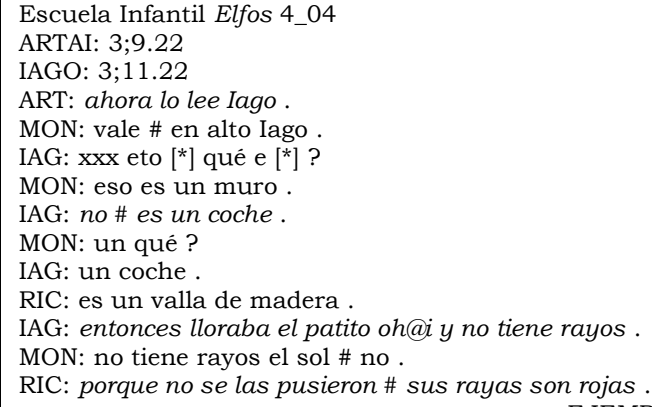


El sentido, la congruencia y la fluidez en la comunicación son elementos esenciales para lograr eficacia. El modo de reaccionar y los tipos de respuesta constituyen indicadores claros del grado de efectividad en las interacciones. El caso de Víctor en el ejemplo 7 (de tres años recién cumplidos) es, a este respecto, muy significativo ya que conversa sobre los dibujos y precisa los objetos (aclara que "no es un palo", sino "un tronco de un árbol"): además de pedir aprobación y reclamar intervenciones ("más rápido que la excavadora, ¿no?”). Resultan asimismo llamativas las reparaciones, e incluso correcciones, sobre algunas apreciaciones que han sido emitidas por sujetos adultos participantes ${ }^{7}$. Sucede en situaciones como la de la muestra 8 , cuando Tatiana (que no ha cumplido aún los tres años) se reitera en que el dibujo observado en la viñeta es un martillo (dadas las similitudes en los trazos de los perfiles de un martillo y de un buzón en casas de campo americanas).

\begin{tabular}{|c|c|}
\hline Escuela Infantil Sta. Susana 5_08 & Escuela Infantil Vite $3 b-02$ \\
\hline MARTÍN: 3;0.17 & TATIANA:2;8.29| \\
\hline VÍCTOR: 3;1.05 & PIL: y aquí, qué es esto? \\
\hline ANI: un palo \# en la trompa \# claro. & PIL: un $+/ /$. \\
\hline VIC: $n o \#<e s>[/]$ es un tronco \# pero de un árbol \# & TAT: ++ martillo. \\
\hline que se rompió [*] \# que lo cortaron $y<v e>[/ /]$ venió & PIL: un qué \\
\hline [*] el elefante $<y$ lo $>$ [/] y lo atrapalló@c. & TAT: martillo. \\
\hline ANI: claro & PIL: ah un martillo! \\
\hline MRT: con la nariz & PIL: no, es un buzón para dejar las cartas. \\
\hline ANI: con la nariz \# que se llama trompa & TAT: no es un busón [*]. \\
\hline MRT: zí [*]! & PIL: no? \\
\hline VIC: <que> [/] que fue más rápido [*] <que> [/] que & PIL: qué es entonces? \\
\hline la excavadoda [*]\# no? & TAT: un martillo. \\
\hline EJEMPLO 7 & EJEMPLO 8 \\
\hline
\end{tabular}

Hay incluso ocasiones en las que se observan anticipaciones a circunstancias previstas o a acciones que se comentan. Y quizás con objeto de evitar tareas o juegos que disgustan. Las excusas y explicaciones elaboradas muestran la capacidad de mentir a través del lenguaje en etapas tempranas. Jorge (de apenas 3 años en el ejemplo 9) recrea una situación pasada en la que hubo de levantarse para justificar ahora el no estar sentado. O cómo Xacobo (de más de tres años en el ejemplo 10) elabora el pretexto de que la caja no se abre para no dejársela a Xulián.

\begin{tabular}{|l|l|}
\hline Escuela Infantil Vite 4_O1 & Escuela Infantil Vite 4_O1 \\
JORGE: $3 ; 2.26$ & XACOBO: $3 ; 7.30$ \\
XULIÂN: $3 ; 1.16$ & XULIÁN: $3 ; 1.16$ \\
ANC: y calladitos eso es y sentaditos. & XUN: <cuando era pequeño me dejo solito mi papi y \\
XUN: muy calladitos. & mi mami y lloré> $[<]$. \\
\hline
\end{tabular}

\footnotetext{
${ }^{7}$ Las reparaciones y reformulaciones son elementos clave en el ajuste de la eficacia de los mensajes, y en mayor medida en edad infantil. Se trata de aproximar los estados mentales de los participantes en la interacción. Según Bernicot, Salazar \& Veneziano (2006:39), "dans une interaction, en particulier entre deux locuteurs qui n'ont pas le même degrè d'expertise du moyen de communication utilisé, il devient pertinent de déterminer les fonctions des énoncés $\mathrm{du}$ point de vue interpsychologique et non plus uniquement du point de vue de l'intention du locuteur".
} 
ANC: claro y sentaditos no se puede uno levantar en el cine o sí?

XUN: no.

XUN: todos muy sentados.

JOR: pues yo fui a_ver tarzán y me levanté um@i porque me iba a sentar bien

$$
\text { EJEMPLO } 9
$$

XAC: yo no lloro porque yo soy más listo que tú. ANC: y te dejaron solo (.) en dónde?

XAC: en el circo.

XUN: me dejas esto?

\%com: Xulián le pide a Jorge el bote de guisantes que tiene en las manos

XAC: no, eso no se abre

$\%$ com: se refiere al bote

EJEMPLO 10

Está claro que las respuestas a la comunicación ilustran estrategias verbales que, aun siendo básicas, son síntoma de participación activa en marcos de intercambio.

\subsection{INTERACCIÓN Y CONVERSACIÓN}

El papel activo del niño en la comunicación se refleja a través de su participación en conversaciones. Es importante observar en primer lugar los hábitos de conversación: con quién lo hace, si es quien inicia y monitoriza la interacción y si los mensajes resultan inteligibles para el interlocutor. En el ejemplo 11, Xacobo (sin llegar a los tres años) es quien plantea una conversación entre iguales, con temática definida, con intenciones apelativas y con cierta carga de empatía al invitar a café a su compañera Tatiana. Logra mantener el tono hasta el extremo de dar explicaciones al margen a adultos que no intervienen directamente en ese intercambio. En ciertas circunstancias, puede que todos los niños participen e incluso quieran iniciar la conversación, el encadenamiento colaborativo es común siempre y cuando la temática resulte atractiva, como sucede en el ejemplo 12. Sin embargo, no siempre se tiene la capacidad para mantener el hilo de su tema, sólo Juan (de tres años y medio lo consigue).

\begin{tabular}{|c|c|}
\hline Escuela Infantil Vite 3b 02 & Escuela Infantil Breogán 604 \\
\hline Xacobo: $2 ; 10.27$ & ELIA: $3 ; 1.09$ \\
\hline Tatiana: $2 ; 8.29$ & JUAN: 3;5.06 \\
\hline PIL: qué tomas? & XAQUIIN: 3;2.22 \\
\hline TAT: leche. & XAQ: pue $[<][/]$ pue yo fui a un cine que habia cineis \\
\hline PIL: leche y qué más. & [*] . \\
\hline $\begin{array}{l}\text { XAC: tú tomas café comigo [*] eh@i Tatiana. } \\
\text { XAC: tú tomas café en el bar comigo [*]. }\end{array}$ & $\begin{array}{l}\text { ANC: fuiste a un cine que habia [//] en el que habia } \\
\text { cisnes? }\end{array}$ \\
\hline PIL: en el bar? & *JUA: pero yo fui a [/] a [/] yo tengo la película de una \\
\hline XAC: sí toma café comigo [*] en el bar. & prinsesa que se convierte en un cirne $\left[{ }^{*}\right]$ ! \\
\hline PIL: os vais a desayunar juntos al bar o qué! & ANC: y nos la cuentas [>] ? \\
\hline $\begin{array}{l}\text { XAC: mira es porque tenemos pega(d)o en la pared } \\
\text { el bal [*]eh@i. }\end{array}$ & $\begin{array}{l}\text { PAU: que yo fui a un acuario y una }<x x x>[>] \text {. } \\
\text { JUA: y habia un }<\text { malo }>[<][>] \text {. }\end{array}$ \\
\hline PIL: ah eso es el bar! & JUA: y habia un malo $<$ Ana $>$ [>]! \\
\hline $\begin{array}{l}\text { \%com: se refiere a algo que está en la habitación } \\
\text { *XAC: no, lo que tenemos pega(d)o en la pared. }\end{array}$ & $\begin{array}{l}\text { ANC: a ver [/] a ver \# estaba hablando Juan \# vamos } \\
\text { a escuchar a }<\text { Juan }>[>] \text {. }\end{array}$ \\
\hline \%act: el niño señala hacia la pared & $\begin{array}{l}\text { JUA: } y \text { habia un [/] y habia un malo en }[/] \text { con la prin- } \\
\text { cesa. At era [?] un baile y [/] y [/] y [/] y le hicia [*] }\end{array}$ \\
\hline EJEMPLO 11 & $\begin{array}{c}\text { bailar con el prin(cipe) [//] con el malo. } \\
\text { EJEMPLO } 12\end{array}$ \\
\hline
\end{tabular}


Asimismo, además de si inicia el intercambio, resulta significativo comprobar, en segundo lugar, cómo mantiene la conversación, si respeta los turnos, si repara el discurso, o si pide aclaraciones. En el fragmento del ejemplo 13, y con apenas dos años y medio Xulián y tres años Xacobo, ambos colaboran en describir una situación e incluso se interpelan para reparar un equívoco.

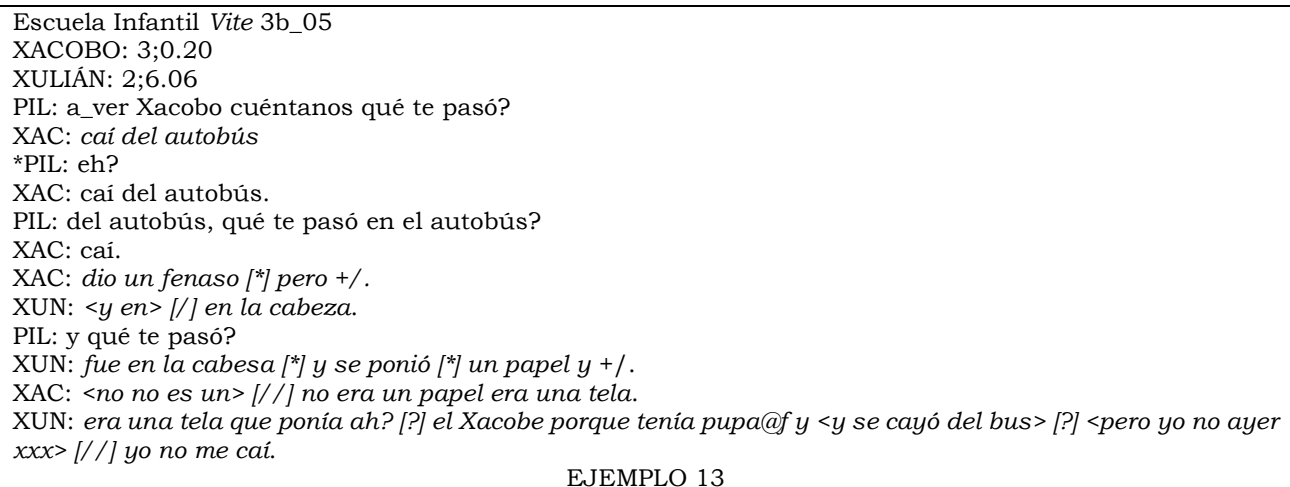

Un caso como el de Artai (ya rozando los 4 años) que comentamos a continuación (ejemplo 14) es revelador del grado notable de efectividad que se logra mediante el manejo de recursos conversacionales como: (a) mantener el hilo y las intenciones a pesar de las interrupciones (parad un poco/parad un poco) y con el propósito de hacer ver las propiedades energéticas del brebaje; (b) reparaciones (no, las culebras no); (c) demanda atención, reclama cooperación e incluso introduce a Ricardo en la trama ( $y$ Ricardo y yo cruzamos); (d) lidera turnos y opiniones (matiza que el bebedizo no es efectivo para vencer las picaduras de serpientes): y (e) resuelve dando un giro al tema (introduce la catarata como motivo de interés en la conversación).

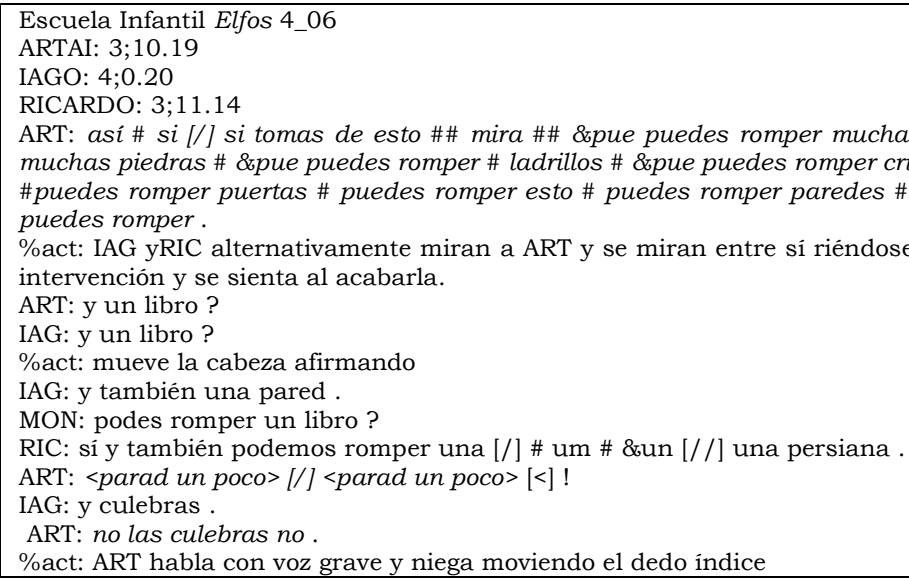


ART: no porque las culebras \# sabes por onde andan \# $x x x$ las pisa le [/] le y [/] y le picó aquí en la boca y después tuvo.

RIC: ja \# pues le piso la cabeza .

ART: pero no pero [/] pero <te entran $>$ [/] te entran <por aqui $>$ [/] por aqui $y$ te van por la barriga $y$ te pican en la barriga y después te [/] te mueres \#\# pero mira.

ART: $x x x$ le [*] hago una patada y va hasta el río y se ahoga .

ART: $y$ se ahoga \# sale [/] sale [/] y sale por $x x x$ y Ricardo $y$ yo $<\mathrm{xxx}>[>], y$ Ricardo $y$ yo cruzamos $y$ sales del río.

RIC: ah@i .

ART: $y$ sale del rio \# y la culebra se va por < una catarata $>[/]$

EJEMPLO 14

Hay, por último, algunos aspectos que denotan la frecuencia de participación y quizás el grado de consciencia sobre normas sociales. Son ilustrativas en este sentido cómo entran, cómo se desenvuelven y cómo cierran sus intervenciones en el discurso; o cuáles son sus reacciones en el marco de conversaciones a las que asisten como oyentes. El inicio de conversaciones o la introducción de nuevos hilos obedecen a motivaciones diversas. Así, Xacobo (con solo dos años y medio en el ejemplo 15) rompe normas cuando se refiere al vino como bebida en el desayuno. En el ejemplo 16 son Jorge (de dos años): Xacobo (de dos años y medio) y Tatiana (de apenas dos años) los que ofrecen esta riqueza en el marco compartido. En esa participación conjunta habitual, pueden respetarse los turnos en una cadena de preguntas y respuestas en la que cada uno desempeña un papel.

\begin{tabular}{|l|l|}
\hline Escuela Infantil Vite 3b_02 & Escuela Infantil Vite 3b_02 \\
TATIANA: $2 ; 3.26$ & TATIANA: $2 ; 3.26$ \\
XACOBO: $2 ; 5.24$ & XACOBO: $2 ; 5.24$ \\
JORGE: $2 ; 0.20$ & JORGE: $2 ; 0.20$ \\
PIL: mamá (.) e que máis tomas? & XAC: por qué corre ese niño? \\
XAC: zumo. & \%com: se refiere al dibujo de un niño que está pegado en la pared \\
XAC: café. & JOR: y porque viene el lobo detlás [*]. \\
PIL: café e que máis? & TAT: por ahí. \\
XAC: vino. & XAC: por qué está el lobo ahí detrás. \\
PIL: vino pola mañá? & JOR: hay una selpiente [*]. \\
PIL: a_ver tú que tomas para de- & TAT: dónde? \\
sayunar Tatiana? & XAC: la flor aquella. \\
JOR: $<y o$ no tomo vino> [>]. & \%com: XAC señala con el dedo hacia la flor que hay en la pared \\
TAT: <vino no> $[<]$. & JOR: <y de alli para acá no ves el lobo> [?]? \\
\multicolumn{2}{|c}{ TAT: ++ (ár)boles. } \\
\hline
\end{tabular}

Está claro que el desarrollo progresivo de habilidades comunicativas corre parejo al grado de participación y desenvoltura por parte del niño en interacciones variadas. La diversidad discursiva y la variedad de intervenciones ante distintos interlocutores son pautas de referencia para valorar grosso modo el grado de eficacia comunicativa en un caso particular. Resultan llamativas las estrategias de Artai (sin haber cumplido aún los 4 años) para narrar, mantener la atención y detentar el liderazgo en el ejemplo $17^{8}$.

${ }^{8}$ G. Prego (2004: 116) señala a este respecto: "Las habilidades pragmáticas que presenta este participante, como jugar con los tonos para contraponer el espacio del narrador al espacio narrado o la manipulación interactiva que hace de la narración para controlar el turno, son más importantes en el liderazgo interactivo de Artai que las estructuras que utiliza”. 
Escuela Infantil Elfos 4_06

ARTAI: $3 ; 10.19$

IAGO: $4 ; 0.20$

RICARDO: $3 ; 11.14$

ART:os voy a contar lo de la catarata \# os voy a contar lo de la catarata \# y la catarata está <todo blanco> [/] todo blanco \# mira y los voy a enseñar \# como esto \# ves \# como esto que es blanco \# ves.

RIC: $y$ como esto también la catarata.

IAG: xxx ahí está estropeado \# ahí un poco \# está estropeado \# ahí .

ART: pero mira \# escoitar \# mira \# y más como esto \# la catarata \# blanca \# mira \# esto es blanco xxx mucho blanco \# hace mucho blanco \# hace mucho blanco .

\%com: RIC y IAG están jugueteando y yo no hacen caso. ART corre detrás de ellos mientras habla porque ya no le prestan atención.

ART: Yago \# Yago \# escucha \# escucha.Yago \# Yago \# te voy a contar lo de la catarata \# pero no me estáis escoitando .

ART: Yago [/] Yago [/] Yago \# escucha [/] escucha lo de la catarata \# escucha \# mira .

ART: y la y después ya no se puede salir de alli, nadie puede salir.

IAG: y por qué?

ART: porque no, porque está todo blanco, y lo moja todo [/] todo [/] todo .

ART: no puede salir.

EJEMPLO 17

En resumen, los modos de interaccionar y de conversar muestran disposiciones clave en la relación entre participantes, señalando su actitud y las previsiones de éxito en sus intervenciones. Así, en el ejemplo 13, si bien Xacobo domina los turnos y las intervenciones, sin embargo, se ve desplazado en su protagonismo en el manejo de recursos socio-comunicativos por Xulián en el momento de describir su accidente en el autobús (era una tela que ponía el Xacobe porque tenía pupa y se cayó del bus). Artai (ejemplos 14 y 17): por su parte, es hábil en su regulación de la conversación ya que siendo líder e imponiendo su turno, permite la cooperación de los participantes a pesar de las burlas (sutilmente, integra a Ricardo en su transacción) (parad un poco, parad un poco; $y$ se ahoga, sale, sale, $y$ sale por xxx $y$ Ricardo y yo, y Ricardo y yo cruzamos y sales del río).

\subsection{VARIACIÓN CONTEXTUAL}

Las estrategias de interacción suelen ajustarse a los parámetros contextuales que las envuelven en aras de conseguir la efectividad comunicativa pretendida. Hay al menos tres dimensiones esenciales con función estelar en la diversidad comunicativa y de registros en el periodo infantil. A saber, (a) los interlocutores, (b) los entornos, y (c) los temas de conversación.

Comunicarse con otras personas y no solo con los familiares, interaccionar con otros niños, o cultivar la conversación con varios interlocutores al tiempo son indicios de un conveniente desarrollo hacia la eficacia. $\mathrm{Si}$, además, los intercambios se producen en ambientes variados no limitados a casa o a la escuela y con temas de conversación que permiten la inclusión de voces narrativas o de similes (por ruido) de objetos, entonces los recursos parecen suficientes para prever osadía y efectividad en la comunicación. En esta misma línea, en el ejemplo siguiente (ejemplo 18): Breixo (de 4 años recién cumplidos) manifiesta destrezas narrativas suficientes mediante el 
uso de voces que dan entrada a los distintos personajes del cuento de Blancanieves.

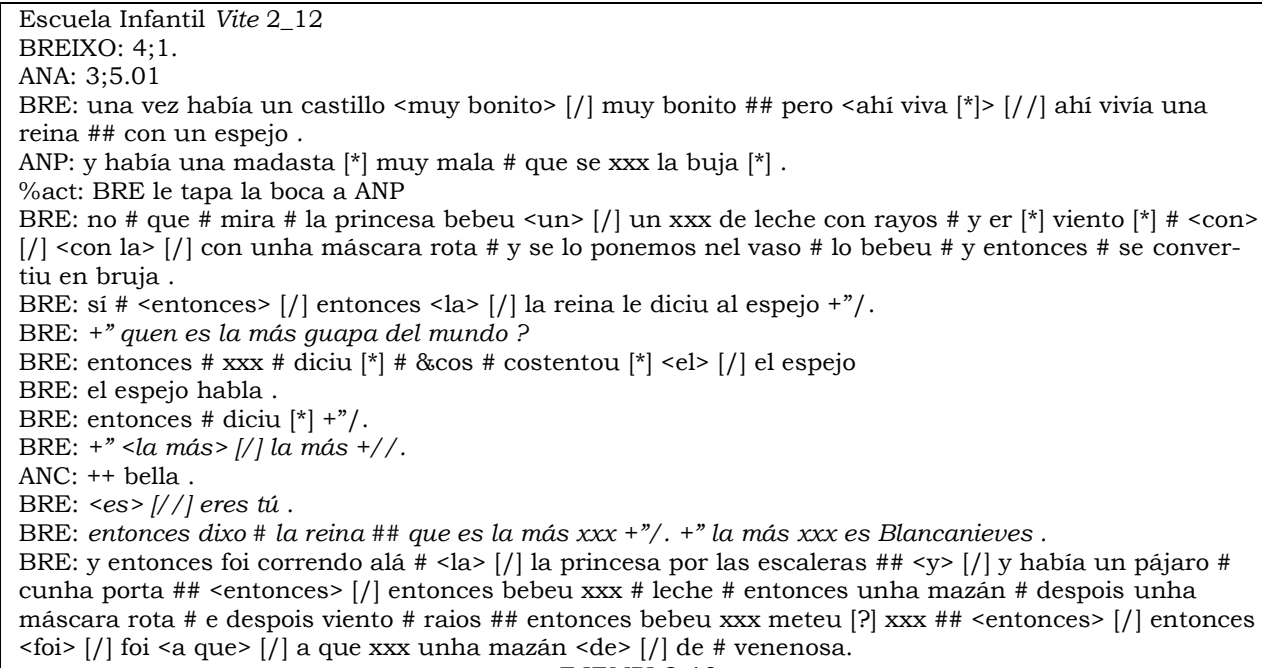

La variación contextual de los actos comunicativos en el habla infantil permite comprobar la riqueza de tácticas de interacción y el dominio de convenciones acordes con el marco. Desde detonantes expresivos como pueden ser libros, juguetes o dibujos, pasando por conversaciones dirigidas en el ámbito familiar o en la escuela, hasta llegar a marcos comunicativos novedosos en donde el niño hace patentes sus habilidades sociales para reconocer intenciones y muestra sus recursos lingüísticos para manifestar sus propósitos. Pueden utilizar lenguaje escatológico sin eufemismos pretendiendo la simpatía, como Xacobo (sin haber llegado a los 4 años, ejemplo 19). O pueden recurrir al circunloquio y al lenguaje indirecto para eludir mencionar la muerte, como lo hacen AnaP (de 3 años y medio) y Breixo (ya de 4 años) en el ejemplo 20.

\begin{tabular}{|l|l|}
\hline Escuela Infantil Vite 4_07 & Escuela Infantil Vite 3a_04 \\
XACOBO:3;11.28 & ANAP: 3;7.22 \\
XULIÁN:3;5.14 & BREIXO: 4;3.21 \\
XUN: brutus <es> [/] es un malo que & ANP: también mi madre trabaja en casa <y en el cole> [>]. \\
está en los dibujos de po(peye) (.) & BRE: <mi papá> [<] [/] mi papá <trabaja> [/] trabaja curando \\
XUN: <y el tiburón> [/] el tiburón & vacas. \\
<que no tie(ne)> [//] que le quitaron & PIL: ah ? verda(de) que teu papá é veterinario (.) non me acordaba \\
los dientes comió el calzoncillo de & eu! \\
brutus. & PIL: y tu papá ? \\
XUN: sí le comió los pantalones y es- & ANP: él tabaja [*] muy lejos. \\
taba en calzoncillos. & PIL: muy lejos muy lejos [!]. \\
XAC: y estaban cagados. & ANP: sí +/. \\
XUN: estaba en calzoncillos. & BRE: trabaja en el cielo decías verdad? \\
\%com: a los niños les hace mucha & ANP: sí (.) hasta arriba de todo. \\
gracia y no paran de reirse & BRE: <trabajaba> [/] trabajaba en el cielo con unas escaleras. \\
XAC: y estaba caga(d)o. & ANP: sí [!]. \\
\multicolumn{2}{|c}{ EJEMPLO 19 }
\end{tabular}


La variación lingüística, en consonancia con los contextos que envuelven los actos verbales, señala el grado de percepción y de asunción del yo en diferentes circunstancias y con el fin de adecuar eficazmente el nivel de interacción. Los fragmentos aducidos revelan las destrezas de Breixo (ejemplo 18) y de Xulián (ejemplo 19) en sus repertorios verbales. El primero incluso puede cambiar de código (gallego y español) dependiendo del tema o del interlocutor. Xulián muestra a veces reflexión metalingüística y valores sociales al reconocer el género gramatical como sintomático del sexo, y es capaz de matizar, reparar y reconducir sus intervenciones sin cambiar significados en una conversación con un adulto, como se constata en el ejemplo 21 :

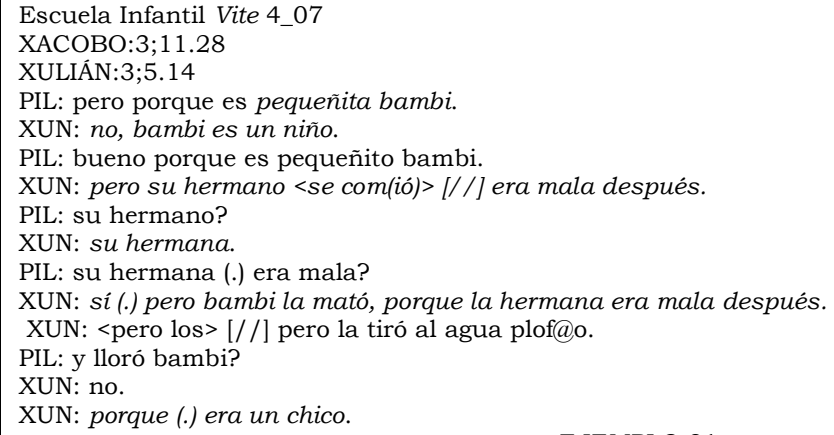

\section{EFICACIA COMUNICATIVA Y EVALUACIÓN DEL LENGUAJE INFANTIL}

Valorar la efectividad previsible de las interacciones infantiles significa considerar la presencia (o la ausencia) de (a) las intenciones comunicativas, (b) la reacción y la respuesta a los intercambios, (c) la participación en conversaciones, y (d) las tácticas comunicativas en distintos contextos. Estos parámetros no son sino señales que prevén la adecuación y la eficacia (Dewart \& Summers 1988; Givon 2002). Un niño cuyas producciones lingüísticas contengan ingredientes ajustados a dichos marcos reunirá condiciones elementales para ser evaluado en sus habilidades comunicativas. Aunque la medida concreta y específica de sus destrezas requiera de los modos estructurales y organizativos que utiliza para elaborar sus mensajes, está claro que, si no se dispone de materiales discursivos y muestras de habla espontánea ponderables en sus significados compartidos, no tiene sentido la evaluación de los procedimientos constitutivos y gramaticales. Únicamente los marcos comunicativos facilitan la consideración de patrones funcionales. De ahí la importancia de recabar emisiones naturales de habla infantil antes que limitar la obtención de datos a cuestionarios formales cerrados en sus respuestas y valoraciones ${ }^{9}$. El habla infantil gira en torno a la dinámica de

\footnotetext{
${ }^{9}$ Los sistemas estandarizados que derivan la evaluación de cuestionarios cerrados no se
} acomodan por lo general al habla genuina y natural de los niños, así que deben sustituirse o 
adquisición, lo importante es el proceso antes que el producto: hay que comprobar y testimoniar si el niño se comunica, si tiene interés por hacerse entender.

Si los marcos sociopragmáticos que hemos subrayado son pautas imprescindibles para valorar niveles basales de eficacia comunicativa, hay características estructurales de la gramática infantil que se vuelven esenciales para medir y evaluar de modo particular el grado de éxito conseguido mediante los recursos expresivos. De algún modo, considerar las habilidades de interacción significa valorarlas en las estrategias comunicativas empleadas, y ello al margen de que su evaluación y ponderación pida entrar en el detalle de rasgos gramaticales acordes, eso sí, con las funciones verbales previstas (Fernández López \& Prego Vázquez 2014). No hay que olvidar los requisitos de ajuste y concordancia entre contornos de praxis y estructuras funcionales. De poco servirá el dominio de reglas sintácticas si no se compadecen con los principios de uso práctico de la lengua ${ }^{10}$.

A este respecto, algunas cuestiones cruciales interconectadas deben ser consideradas. En primer lugar, parece necesario notar qué elementos estructurales son imprescindibles en las emisiones verbales para poder hablar de eficacia. De hecho, hay opciones teóricas que abordan el proceso de desarrollo desde un enfoque social y comunicativo y reconocen porciones cognitivas esenciales categorizadas en elementos lingüisticos estructuralmente imprescindibles. Predicaciones básicas como las señaladas por Brown (1973) para referenciar el mundo, los mensajes-palabra en un golpe de voz en las emisiones orales (las denominadas holofrases): las dinámicas del item-a-lasconstrucciones paulatinamente más complejas propuestas por Tomasello (2003): o los procesos construccionales básicos como la predicación, la combinatoria y la concordancia (Fernández Pérez, 2006a; Gallardo Paúls, 2009): reseñan unidades y procedimientos exclusivos del habla infantil necesarios para el progreso de una práctica comunicativa eficaz. Según Bowerman \& Levinson (2001:13): las investigaciones de corte interlingüístico, y que comparan procesos de desarrollo en lenguas muy diversas, revelan percepciones cognitivas universales plasmadas en porciones semánticas generales y básicas en el proceso de adquisición de cualquier lengua. Basta con prestar atención al habla infantil sin condicionantes impuestos del lenguaje adulto:

On the linguistic side, it is easy to show that languages diverge in the way they classify various domains, and it has been shown that, from very earliest speech, children are closer to their adult targets than to children speaking other contrasting languages (...) A problem with the analysis of children's early language is that we often cannot be sure

complementarse por medio de procedimientos cualitativos que permitan valorar el discurso. Las baterías y las pruebas que sopesan el habla infantil deben respetar las características singulares de los procesos y no pueden perder de vista su naturaleza evolutiva (Fernández Pérez, 2005).

${ }^{10}$ Las producciones en casos de sindrome de Williams constituyen el ejemplo más palmario de esas exigencias de equilibrio entre el dominio de estructuras formales y las tácticas para ser eficientes en interacciones comunicativas. 
that we understand their own immature semantics: perhaps the early language usage is less in line with adult targets than it seems.

Además, en segundo lugar, conviene plantearse si esas propiedades elementales hay que aislarlas por componentes autónomos o si resulta más apropiado hacerlo de modo integral. En la actualidad no se pone en duda que el habla infantil contiene propiedades y rasgos genuinos que no son equivalentes a las características del lenguaje adulto. Hay aspectos en el habla de los niños que la definen como peculiar y que le confieren entidad y sentido propio en los intercambios comunicativos (Peters 1983; Tomasello 2003; Clark 2003, 2016). Los enfoques teóricos más tradicionales, anclados en la lengua como producto ya configurado y sobre un modelo de lengua escrita, subrayan propiedades por cada uno de los componentes. Por su parte, las aproximaciones a los usos, que conciben la lengua en proceso en una dinámica emergente hacia la comunicación, realzan de manera global las singularidades insistiendo en su dimensión significativa. Como es natural, la eficacia en la interacción se define por las repercusiones de los usos verbales y por el grado de incidencia de los mensajes, así que lo pertinente parece ser la aproximación global e integrada. No importa tanto que las muestras de habla del niño contengan procesos fónicos propios de cada etapa ([kóke] y no [kóce], [tí] y no [sí], o [lópe] y no [rómpe]): o que los esquemas argumentales se limiten a hormas semánticas elementales (como temarema en "a mí tero", o como posesión en "mío libo"): cuanto que las producciones vayan cargadas de contenido en contextos y situaciones. Finalmente, en tercer lugar, cabe preguntarse por la previsible y natural relevancia del prisma evolutivo en la determinación de tales rasgos formales soporte de la eficacia. Si bien en perspectiva evolutiva es esperable un incremento en complejidad y riqueza construccional del habla infantil, sin embargo, no siempre tiene que ir acompañado de una progresión proporcional en el logro de efectividad comunicativa. Ni la riqueza gramatical ni el caudal léxico son condiciones suficientes para la eficacia, se hace necesario ponderarlas en su ajuste a la praxis comunicativa. La adecuación a contextos e intenciones de los usos sistemáticos será determinante para la medida (Hernández Sacristán 2006). Ciertamente, hay ya un número considerable de pruebas evaluativas que organizan las funciones verbales requeridas por niveles de progresión de desarrollo, distribuyéndolas en escalas evolutivas y entrecruzando los componentes pragmático, semántico y sintáctico. De manera que, desde un prisma que integra componentes y contempla globalmente el proceso de adquisición, determinadas características pragmáticas corren parejas a ciertas funciones semánticas y se asocian con esquemas sintácticos específicos. Las escalas de desarrollo de la batería de Bristol (Gutfreund et 
alii 1989) pueden ser una excelente ilustración de esta imbricación de componentes y del ajuste entre funciones paralelas ${ }^{11}$.

Así pues, valorar las habilidades comunicativas de los niños en las distintas etapas de desarrollo y a través de los datos verbales obtenidos de sus actividades de interacción verbal, pide prestar atención a los rasgos reiterados en las muestras que, aun siendo peculiares, dan soporte a codificaciones propias de la gramática infantil (Fernández López \& Fernández Pérez 2014). En las construcciones y procesos propios de la dinámica adquisitiva se sostienen de manera global las cotas de eficacia comunicativa que convienen a las expresiones del habla de los niños. Esa consistencia unitaria e integral explica la trabazón de repercusiones en la dinámica de avance entre componentes y unidades. No sólo porque el desarrollo fónico corre paralelo a la eclosión de las palabras, o porque el caudal léxico regula la activación y la fertilidad de combinatorias construccionales de cierta longitud y complejidad (cfr. Fernández Pérez 2004): sino sobre todo porque la motivación comunicativa y los móviles de interacción van a ser los desencadenantes de la actividad verbal en todas sus dimensiones. Conviene no olvidar que la motivación en el habla infantil es comunicativa y que son detonantes de interacción los que dinamizan el avance de la gramática formal (Prego Vázquez 2004; Enríquez Martínez 2017). De hecho, valorar el éxito comunicativo en el habla infantil equivale a contemplar los intercambios verbales según los cuatro parámetros descritos. De algún modo, los niveles basales de interacción efectiva se hallan en las características mencionadas.

En resumen, determinar el rango de desarrollo de la eficacia requiere combinar las dos dimensiones de los usos verbales -la comunicativa y la formal- en marcos de significado por franjas de edad. Las condiciones esenciales para alcanzar grados suficientes de efectividad se completan con exigencias necesarias de sistematicidad que les confieran soporte codificado en construcciones formales (Fernández López \& Fernández Pérez 2014).

\footnotetext{
${ }^{11}$ Evaluar el grado de desarrollo de los recursos y estrategias que los niños manejan para ser eficaces en sus intercambios exige considerar el soporte estructural asociado a dichas dimensiones de praxis. Bien entendido que las propiedades formales no se precipitan de modo independiente, sino que su complejidad y extensión corre pareja a ciertas funciones pragmáticas y según estadios cronológicos determinados. Así, funciones pragmáticas como la apelación o la señalización se canalizan en formatos verbales sencillos desde las primeras etapas, pero tienen también soporte en estructuras formales más complejas en estadios superiores. Otras funciones como la condición o las fórmulas indirectas parecen propias de fases avanzadas y piden hormas estructurales específicas de niveles altos. En la batería de desarrollo de Bristol, el denominado BLADES (Bristol Language Development Scales, cfr. Gutfreund et alii 1989), las funciones pragmáticas de sugerencia y formulación indirecta (en el nivel VI de la escala de desarro1lo) se correlacionan con esquemas sintácticos complejos que contienen significados gramaticales de obligación / necesidad. La función de condición (en el nivel X, el más elevado en la escala) va de la mano de estructuras de contenido inferencial y premeditado.
} 


\section{Conclusión. La eficacia comunicativa como pauta de desarkollo VERBAL}

Que un niño sea eficaz en sus interacciones significa que utiliza recursos para hacerse entender, que desarrolla estrategias para ser comunicativo en diferentes situaciones y con interlocutores variados. Los indicadores pragmáticos que hemos reseñado en este trabajo son conditio sine qua non para sentar las bases de la eficiencia comunicativa y del progreso en la dinámica adquisitiva.

En conjunto, tales elementos forman parte de la autorregulación del niño ya que delinean su situación respecto de los otros y permiten dibujar lo que Vygotsky (1934) llama conciencia reflexiva. Una prueba más de que la fase egocéntrica proclamada por Piaget (1923): si bien con importancia en el desarrollo psicológico-cognitivo, apenas tiene sentido en la dimensión adquisitiva de la lengua. Desde muy pronto, las emisiones sonoras del niño se acompañan de funciones comunicativas comprendidas por los cuidadores y allegados, de modo que el pretendido egocentrismo ensimismado aparece mitigado por las transacciones alcanzadas y por los mecanismos de atención e imitación muchas veces asociados.

Como se ha constatado, las intenciones contienen, ya en las primeras etapas, la carga directiva de los mensajes. La diversidad de disposiciones anímicas y la emergente personalidad del niño muestran no sólo su escaparate cognitivo-emocional, sino también la gama de recursos elementales para ser efectivos en la interacción. Los ejemplos 3 y 4 ilustran la fuerza transaccional de las emisiones infantiles mediante tácticas de repetición y de cooperación. Tanto Irene como David y Antía logran intencionadamente sus propósitos. Asimismo, las pretensiones puramente comunicativas cargadas de expresividad y sensaciones sobre "la culebra" son manifiestas en el caso de Artai en el ejemplo 5.

Por otra parte, las respuestas a la comunicación hacen patentes estrategias verbales básicas que revelan participación activa en marcos de intercambio. Resulta llamativa la disposición de Iago en el ejemplo 6 a la propuesta de lectura del cuento, así como los detalles de perspicacia sobre las imágenes que describe. También la actitud clarificadora, reparadora y demandante de aprobación de Victor en el ejemplo 7 manifiesta sus habilidades para responder en las coordenadas de intercambio en que se encuentra.

Tal y como se ha evidenciado, los modos de interaccionar y conversar proyectan actitudes clave en la relación entre participantes, señalando su disposición y las previsiones de éxito en sus intervenciones. Así, si bien Xacobo (en el ejemplo 13) domina los turnos y las intervenciones, no obstante, se ve desplazado en su protagonismo en el manejo de recursos sociocomunicativos por Xulián sobre todo cuando trata de describir su accidente en el autobús. Artai, por su parte, es hábil en su regulación de la conversación ya que siendo líder e imponiendo su turno, permite la cooperación de 
los participantes (sutilmente, integra a Ricardo en su transacción, ejemplo 14).

Finalmente, la variación lingüística, en consonancia con los contextos de los actos verbales, señala el grado de percepción y de asunción del yo en diferentes circunstancias y con el fin de adecuar eficazmente el nivel de interacción. Las destrezas de Breixo para dar entrada a voces en el ejemplo 18, y de Xulián para manejar lenguaje escatológico en el ejemplo 19 prueban la riqueza de sus repertorios verbales. El primero incluso puede cambiar de código (gallego y español) dependiendo del tema o del interlocutor. Xulián muestra a veces reflexión metalingüística y valores sociales aprendidos al reconocer el género gramatical como sintomático del sexo en el ejemplo 21 .

En sintesis, estimar la eficacia comunicativa del habla infantil equivale a ponderar dentro de un marco referencial por márgenes de edad las tácticas verbales efectivas para ajustar el sentido del mensaje. Las estrategias de interacción verbal en el habla de los niños resultan comunicativamente exitosas cuando vehiculan significados con sentido mediante estructuras y construcciones propias de las distintas etapas de desarrollo. Es así como la observación y el análisis valorativo en términos de eficacia comunicativa dan entrada al prisma de "¿qué consigue el niño con lo que tiene a su disposición?». Punto de vista éste que promueve el interés tanto por las características singulares que canalizan los contenidos efectivos de los mensajes elaborados, cuanto por aquellos rasgos que oscurecen o imposibilitan el intercambio. Valorar el habla infantil no sólo depara sorpresas por la presencia inaudita de mecanismos gramaticales emergentes con su correspondiente alcance comunicativo, sino que también puede suscitar sospechas respecto de procesos anquilosados o de esquemas construccionales rudimentarios o con escasa determinación.

\section{REFERENCIAS}

ARBIB, M. (2002): “The Mirror System, Imitation, and the Evolution of Language", Kerstin DAUTENHAHN \& Chrystopher NEHANIV (eds): Imitation in Animals and Artifacts, The MIT Press, pp. 229-280.

BERNICOT, J.; SALAZAR, A. \& VENEZIANO, E. (2006): "Les reprises: dialogue, formes, fonctions et ontogenèse", La Linguistique, 42/2, pp. 29-50.

BOWERMAN, M. \& LEVINSON, S. C. (eds.) (2001): Language acquisition and conceptual development, Cambridge: Cambridge University Press.
BROWN, R. W. (1968): "The development of WH-questions in child speech", Journal of Verbal Learning \& Verbal Behavior, 7, 279-290.

BROWN, R. W. (1973): A first language: the early stages, Cambridge: The Harvard University Press.

BRUNER, J. (1983): Child's Talk: Learning to Use Language, New York: Norton. Traduc. al español de R. Premat, El habla del niño. Aprendiendo a usar el lenguaje, Barcelona: Paidós, 1995. 
BUCCINO, G.; BINKOFSKI, F. \& RIGGIO, L. (2004): "The mirror neuron system and action recognition", Brain and Language, 89/2, pp. 370-376.

CLARK, E. V. (2003): First Language Acquisition, Cambridge: Cambridge University Press.

CLARK, E. V. (2006): "La répétition et l'acquisition du langage", La Linguistique, 42/2, pp. 67-79.

CLARK, E. V. (2016): Language in Children, London: Routledge.

CLARK, E. V. \& CHOUINARD, Mi. (2000): "Énoncés enfantins et reformulations adultes dans l'acquisition du langage", Langages, 140, pp. 9-23.

CORBALLIS, M. (2006): "Evolution of Language as a Gestual System", Marges Linguistiques, 11 , 2006, pp. 218-229.

DEUTSCH, W.; WAGNER, A.; BURCHARDT, R.; SCHULZ, N. \& NAKATH, J. (2001): "Person in the language of singletons, siblings and twins", BOWERMAN, M. \& LEVINSON, S. (eds.): Language Acquisition and Conceptual Development, Cambridge: Cambridge University Press, pp. 284-315.

DEWART, H. \& SUMMERS, S. (1988): Pragmatics Profile of Early Communication Skills, Orford: NFER-Nelson.

ENRÍQUEZ MARTÍNEZ, I. (2017): Del discurso a la gramática en el habla infantil. Cómo los marcadores evolucionan a conectores de construcciones complejas, Madrid: Arco Libros.

FERNÁNDEZ PÉREZ, M. (2004): “Adquisición del lenguaje y componentes de la lengua", en Milka VILLAYANDRE (ed.): Actas del V Congreso de Lingüística General (León): Madrid: Arco Libros, pp. 71-89.

FERNÁNDEZ PÉREZ, M. (2005): "¿Cómo evaluar el lenguaje infantil?”, VEYRAT, M. \& SERRA,
E. (eds.): Estudios de Lingüistica Clinica/4, Valencia: Universitat de València, pp. 55-78.

FERNÁNDEZ PÉREZ, M. (2006a): "Hacia una 'gramática' del habla infantil. Enfoques lingüísticos básicos", LUQUE, J. D. (ed.): $V$ Congreso Andaluz de Lingüística. Homenaje a José A. de Molina, Granada: Método, pp. 1299-1315.

FERNÁNDEZ PÉREZ, M. (2006b): "Usos verbales y adquisición de la gramática. Construcciones y procesos en el habla infantil", Revista Española de Lingüística, 36, pp. 319-348.

FERNÁNDEZ LÓPEZ, I. \& FERNÁNDEZ PÉREZ, M. (2014): "Sistemas de valoración del habla infantil", FERNÁNDEZ PÉREZ. M. (coord.): Lingüistica y déficits comunicativos. ¿Cómo abordar las disfunciones verbales?, Madrid: Sintesis, pp. 101-139.

FERNÁNDEZ LÓPEZ, I. \& PREGO VÁZQUEZ, G. (2014): "Exploración lingüistica del habla infantil”, FERNÁNDEZ PÉREZ, M. (coord.): Lingüística $y$ déficits comunicativos. ¿Cómo abordar las disfunciones verbales?, Madrid: Sintesis, pp. 45-99.

GALLARDO PAÚLS, B. (2009): "Criterios lingüísticos en la consideración del déficit verbal", Verba, 36, pp. 327-352.

GIVON, T. (2002): “The visual information-processing system as an evolutionary precursor of human language", GIVÓN, T. \& MALLE, B. (eds.): The Evolution of Language Out of Pre-language, Amsterdam / Philadelphia: John Benjamins Publishing Company, pp. 3-50.

GIVON, Th. (2009): The Genesis of Syntactic Complexity: Diachrony, Ontogeny, Neuro-Cognition, Evolution, Amsterdam: J. Benjamins. 
GIVON, T. \& MALLE, B. (eds.) (2002): The Evolution of Language out of Pre-language, Amsterdam: John Benjamins.

GUTFREUND, M.; HARRISON, M. \& WELLS, G. (1989): Bristol Language Development Scales, Oxford: NFER-Nelson.

HERNÁNDEZ SACRISTÁN, C. (2006): Inhibición y lenguaje. A propósito de la afasia y la experiencia del decir, Madrid: Biblioteca Nueva.

LEVELT, W. J. (1983): "Monitoring and self-repair in speech", Cognition, 14, pp. 41-104.

OCHS, E. \& SCHIEFFELIN, B. (1995): "The Impact of Language Socialization on Grammatical Development", FLETCHER, P. \& MACWHINNEY, B. (eds.): The Handbook of Child Language, Oxford: Blackwell, pp. 73-94.

PETERS, A. (1983): The units of language acquisition, Cambridge: Cambridge University Press.

PIAGET, Je. (1923): Le langage et la pensee chez l'enfant, Paris: Édit. Delachaux \& Nestle. Traducción al español de M. Riani, El lenguaje $y$ el pensamiento en el niño, Buenos Aires: Guadalupe, 1983.

PREGO VÁZQUEZ, G. (2004): "Las narrativas en niños de tres/cuatro años: un puente entre la sintaxis y la pragmática", en Montserrat VEYRAT \& Beatriz GALLARDO (eds.): Estudios de Lingüística clinica/3. Aspectos evolutivos, Valencia, Universitat, pp. 99-126.
RIZZOLATI, G. \& ARBIB, M. (1998): "Language within our grasp", Trends in Neurosciences, 21, pp. 188-194.

TOMASELLO, M. (2003): Constructing a Language: A Usage-Based Theory of Language Acquisition, Harvard University Press.

TREVARTHEN, C. (1980): "The Foundations of Intersubjectivity: Development of Interpersonal and Cooperative Understanding in Infants", en David OLSON (ed.): The Social Foundations of Language and Thought, New York: W.W. Norton \& Co., pp. 316342.

TREVARTHEN, C. (1993): "The Self Born in Intersubjectivity: The Psychology of an Infant Communicating", NEISSER, U. (ed.): The Perceived Self, Cambridge University Press, pp. 121-173.

TREVARTHEN, C. (1998): "The concept and foundations of infant intersubjectivity", BRATEN, S. (ed.): Intersubjective Communication and Emotion in Early Ontogeny, Cambridge: Cambridge University Press, pp. 15-46.

VYGOTSKY, L. (1934): Myshlenie $i$ rech, Mockba. Traducción al español de P. Tosaus Abadía (sobre la edición inglesa a cargo de A. Kozulin, Thought and language, Cambridge, The MIT Press, 1986): Pensamiento $y$ lenguaje, Barcelona: Paidós, 1995. 